\title{
Reconstruction of iliac crest defect after autogenous harvest with bone cement and screws reduces donor site pain
}

\author{
Jing Zhang ${ }^{1 \dagger}$, Yuxuan Wei $^{1 \dagger}$, Yue Gong ${ }^{2}$, Yang Dong ${ }^{1 *}$ and Zhichang Zhang ${ }^{1 *}$ (D)
}

\begin{abstract}
Background: The iliac crest is the most common autogenous bone graft donor site, although associated with postoperative pain, functional disability, cosmesis, morphology and surgical satisfaction. We assessed each aspect above by comparing iliac crest reconstruction with bone cement and screws following harvest with no reconstruction.

Methods: We evaluated patients who underwent large iliac crest harvesting, including ten patients who underwent iliac crest defect reconstruction with bone cement and cancellous screws (R group) and ten randomly matched patients without reconstruction (NR group) were evaluated prospectively in the same period. Local pain, cosmesis and other complications were assessed postoperatively at 1 week, 6 weeks, 3 months and 6 months.

Results: Pain, cosmesis and satisfaction of patients significantly differed between the two groups. The $R$ group exhibited less complications and lower pain visual analogue scores at postoperative 1 week $(p<0.001), 6$ weeks $(p<0.001)$ and 3 months $(p<0.01)$ but not at 6 months, at which time patients reported almost no pain. One patient reported pain for more than 1 year in the NR group. The R group exhibited better cosmesis, morphology and satisfaction than the NR group. In the NR group, one patient suffered pain when sitting up and another when wearing a belt.

Conclusion: Postoperative pain can be reduced and cosmesis can be improved through reconstructing the iliac crest defects after autogenous harvesting with bone cement and cancellous screws. The technique is simple, safe and easy to implement.
\end{abstract}

Keywords: lliac crest bone graft, Morbidity, Reconstruction of donor site, Bone cement

\section{Background}

Autogenous bone grafts are widely used in clinical orthopedics due to their biological and nonimmunologic properties compared with other materials. The iliac crest is the most common donor site because of its easy access, low morbidity and ability to provide sufficient quantities of both cortical and cancellous bone [1]. However, complication rates following iliac crest bone harvesting have been reported from 2 to $49 \%$. Most patients suffer from pain which has an effect on sleep within 1 month after surgery, and even 13 to $20 \%$ of the

\footnotetext{
* Correspondence: dongyang6405@163.com; zzc_sjtu@sjtu.edu.cn

${ }^{\dagger}$ Jing Zhang and Yuxuan Wei contributed equally to this work.

'Department of Orthopaedic Surgery, Shanghai Jiao Tong University

Affiliated Sixth People's Hospital, Shanghai 200233, China

Full list of author information is available at the end of the article
}

patients experience chronic pain [2, 3]. The most common complication is pain at the donor site after that less frequently complications including secondary fracture, superficial numbness, infection, abdominal hernia and gait abnormality [4-6]. In addition, harvesting a large graft from iliac crest bone leads to some problems such as depression of surgical area, poor cosmetic appearance, having influence on walking, recreation, household chores and so on [2].

Some studies have indicated that reconstruction of iliac crest defects after harvesting can reduce complications [7-10]. Various implantation materials and techniques have been used to rebuild iliac defects. Burton and colleagues [11] reconstructed the iliac crest with a hydroxyapatite-calcium triphosphate

(c) The Author(s). 2018 Open Access This article is distributed under the terms of the Creative Commons Attribution 4.0 International License (http://creativecommons.org/licenses/by/4.0/), which permits unrestricted use, distribution, and reproduction in any medium, provided you give appropriate credit to the original author(s) and the source, provide a link to the Creative Commons license, and indicate if changes were made. The Creative Commons Public Domain Dedication waiver (http://creativecommons.org/publicdomain/zero/1.0/) applies to the data made available in this article, unless otherwise stated. 
biphasic compound, which improved the body's ability to reform new bone but did not alleviate the pain. Furthermore, the technique is only suitable for harvesting parts of cancellous bones. Other studies have been carried out for the repair of iliac crest defects using autogenous bone [7], bovine cancellous grafts [9], polymethyl methacrylate bone cement [12] and allografts fixed with cannulated screws [10]. Although such studies provided some options to reduce the donor site pain, we still try to find a more simple and effective method.

In this study, we used a new technique with bone cement and screws to repair large iliac bicortical bone defects after autogenous harvesting for bone tumors, such as giant cell tumors of bone or other benign tumors. The purpose of this study was to evaluate the benefits of this new method.

\section{Methods}

\section{Study population}

Patients who underwent autogenous iliac crest grafting to repair a bone defect or nonunion after primary bone tumor excision in 2016 were selected for inclusion in this trial. The following inclusion criteria were utilized for patient selection: surgical treatment with a large iliac crest graft for repair of a bone defect or nonunion association with primary bone tumor excision; harvest of more than $40 \mathrm{~mm} \times$ $30 \mathrm{~mm}$ of iliac crest; and patients aged between 18 and 60 years old. Patients with osteoporosis or who could not tolerate the surgery for iliac crest reconstruction were not included. That patients were asked whether they wanted to receive conventional procedures or the new method prior the study took place and that the control group was matched after the study population for the new method was gathered.

Twenty patients were included in this study. Ten patients who conducted reconstruction were allocated to the reconstructed group ( $\mathrm{R}$ group) and were compared with 10 patients whose iliac crest defect was not reconstructed (NR group). All surgical procedures were performed by the same chief surgeon.

\section{Operation technique}

Both groups underwent the same procedure of the harvest. The volume of the bone graft depended on the volume of the tumor. The iliac crest graft harvest was on the same side as the bone tumor. In supine position, a skin incision was made along iliac crest contour. We dissected soft tissue and harvested the required amount of iliac crest using an osteotome from $3 \mathrm{~cm}$ posterior to the anterior superior iliac spine (Fig. 1a). In the $\mathrm{R}$ group, according to the harvesting size, three or more cancellous screws were implanted leaving $2-3 \mathrm{~cm}$ of head end out of the bone as an anchor providing support and adhesion for the bone cement (Fig. 1b). The defect area was filled with bone cement which was formed into the shape of the iliac crest contour (Fig. 1c). Careful attention was paid to ensure that the cement did not extend beyond the original shape of the bone to avoid discomfort. After the bone cement had solidified, a drainage tube was placed, and the incision was closed. The only difference between the two groups was that the defect of the iliac crest was reconstructed in the $\mathrm{R}$ group, whereas no reconstruction was performed in the NR group.

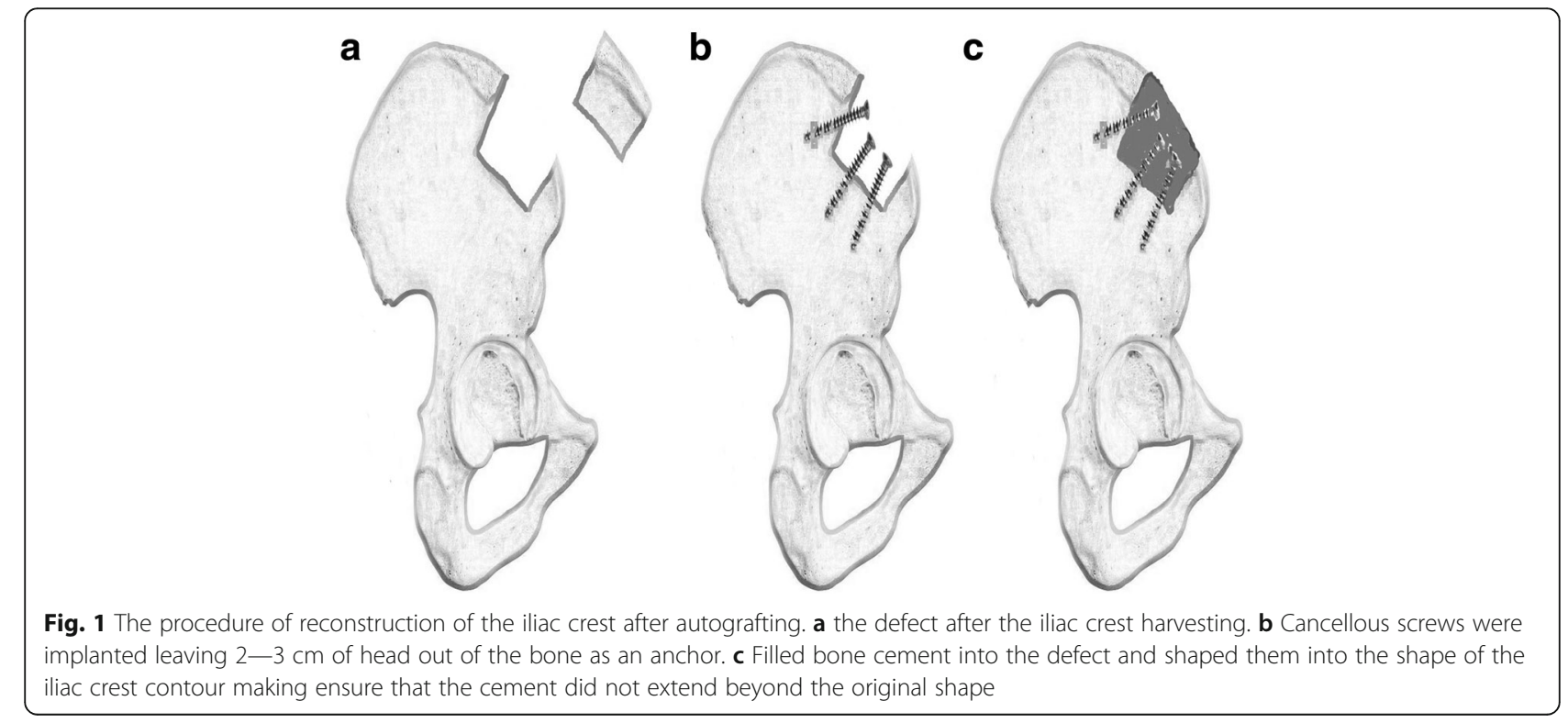


Table 1 The clinical assessment contents and criteria

\begin{tabular}{|c|c|}
\hline Pain VAS & Range of 0 to $10(0$ indicates no pain, 10 indicates the worst pain imaginable) \\
\hline \multirow[t]{3}{*}{ Function disability } & Sitting up \\
\hline & Wearing a belt \\
\hline & Sleep on the operation side \\
\hline $\begin{array}{l}\text { Cosmesis of the operated } \\
\text { side }\end{array}$ & Range of 0 to 10 ( 10 indicates that the appearance is almost no different from the contralateral side. 0 means very bad) \\
\hline \multirow[t]{2}{*}{ Depression of the defect } & The same as the contralateral side (depression $<1 \mathrm{~cm}$, normal) \\
\hline & Depressed compared with the contralateral side(> $1 \mathrm{~cm}$, depression) \\
\hline Surgical satisfaction scale & Range of 0 to 10(10 indicates it's very satisfied. 0 indicates it's not satisfied at all) \\
\hline
\end{tabular}

\section{Assessment}

Postoperative pain was evaluated using the pain visual analog scale (pain VAS) in both groups at 1 week, 6 weeks, 3 months and 6 months after the surgery. Functional disability was evaluated based on the pain of the patients reported when they performed 3 daily activities: sitting up, wearing a belt, and sleeping on the operation side. Cosmesis of the operated site, morphology of the defect, and surgical satisfaction scale (SSS) were only assessed at 6 months (Table 1). Complications were recorded during the follow-up and a radiologic evaluation was performed using posterior-anterior radiograph images of the pelvis at 1 week, 6 weeks, 3 months and 6 months after the surgery.

Differences between groups were analyzed using Student's t-test and Fisher's exact test. All statistical analyses were performed using SPSS 20.0 software (SPSS Inc., Chicago, IL). $P<0.05$ was considered as statistically significant.

\section{Results}

The demographic data between the two groups had no significant differences, as presented in Table 2. The pain VAS of the $\mathrm{R}$ group was $1.1 \pm 0.53$, markedly lower than that of the NR group of $4.3 \pm 0.48(p<0.001)$ at 1 week after surgery. Similarly, the VAS score showed significant differences between the two groups at 6 weeks $(0.3 \pm$ 0.46 vs $1.8 \pm 0.42, p<0.001)$ and 3 months ( 0 vs $0.8 \pm$ $0.63, p<0.01)$. In comparison to the $\mathrm{R}$ group where no patient suffered from pain after 6 months $(p=0.29$, Fig. 2). However, one patient suffered from pain for more than 1 year in the NR group.

Complications in the $\mathrm{R}$ group included one patient who reported thigh numbness and one who reported slight pain when wearing a waist belt during the first
6 months postoperatively, which were alleviated by the sixth month. On the other hand, two patients reported numbness on the outside of the thigh in the NR group. One patient suffered pain when wearing a waist belt and another patient reported ache when sitting up.

There was a significant difference between the two groups in cosmesis assessment. The mean score of cosmesis was 9.6 in the $\mathrm{R}$ group, which was significantly higher than that of the NR group of $7.5(p<0.001)$. The result of SSS was consistent with the cosmesis score (Fig. 3). The morphology of the two groups was also significantly different. In the $\mathrm{R}$ group, there was no depression, whereas 9 cases of depression appeared in the NR group $(p<0.001$, Table 2$)$.

Posterior-anterior radiograph images of the pelvis were assessed postoperatively during the 6 months. There was no secondary fracture of bone or dislocation or fractured bone cement in both groups (Fig. 4).

\section{Discussion}

The iliac crest remains the most frequently site for autogenous grafts with many advantages compared with other sites such as distal radius and artificial bone. However, postoperative pain and poor cosmesis are major deterrents in harvesting bone from the iliac crest. Different methods have been used to solve these problems, such as injection of local anesthetic which have achieved different levels of success $[1,13]$. However, the results were still not satisfying because of the problems mentioned above. Therefore, some researchers have attempted to reconstruct the iliac crest after harvesting bone and discovered that it could alleviate donor site pain and access good cosmesis $[8,10,14,15]$.

In our study, we used bone cement and cancellous screws to reconstruct the defect of the iliac crest after

Table 2 The demographic data of all patients

\begin{tabular}{llll}
\hline & R group & NR group & $P$ values \\
\hline Number of patients & 10 & 10 & $p=0.634$ \\
Age(years) & $38.7 \pm 14.2(18-59)$ & $36 \pm 10.2(24-53)$ & $P=1$ \\
Sex (F:M) & $5: 5$ & $6: 4$ & $p=0.297$ \\
Average size of iliac crest defect $\left(\mathrm{cm}^{2}\right)$ & $14.55 \pm 2.0$ & $13.5 \pm 1.6$ & \\
\hline
\end{tabular}




\begin{tabular}{llcr}
\hline & & R group & NR group \\
\hline The pain VAS & 1 week & $1.1 \pm 0.54$ & $4.3 \pm 0.48$ \\
& 6 weeks & $0.3 \pm 0.46$ & $1.8 \pm 0.42$ \\
& 3 months & 0 & $0.8 \pm 0.63$ \\
& 6 months & 0 & $0.3 \pm 0.67$
\end{tabular}

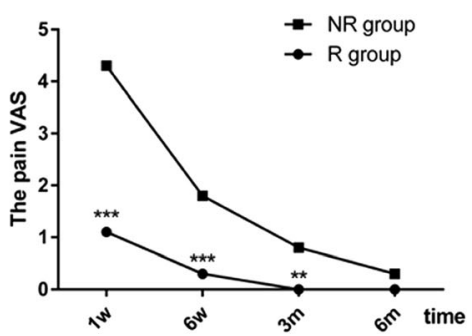

Fig. 2 The pain VAS scores between the two groups during the postoperative 6 months. The pain VAS between the R group and the NR group was $1.1 \pm 0.53$ vs $4.3 \pm 0.48,0.3 \pm 0.46$ vs $1.8 \pm 0.42,0$ vs $0.8 \pm 0.63$ and 0 vs $0.3 \pm 0.67$ at 1 week, 6 weeks, 3 months and 6 months after surgery. ${ }^{* *}$ $p<0.01,{ }^{* * *} p<0.001$

harvesting. The result of this study revealed significant differences between the two groups at postoperative 1 week, 6 weeks and 3 months. No patient experienced chronic pain in the $\mathrm{R}$ group compared with the NR group. The results of other complications, cosmesis and satisfaction in the $\mathrm{R}$ group were better than those in the NR group.

Some researchers reconstructed the iliac crest after autografting using different methods and materials $[10,12]$. Y-F Niu and colleagues shaped the allograft iliac crest to match the defect and reconstructed the anatomical contour, and then they fixed the allograft with hollow compression screws [10]. Jong Seong Lee and colleagues harvested a wedge of iliac crest with a bone burr on each wall and filled the defect with bone cement [12]. The results of theirs were similar in postoperative pain, cosmesis and additional complications. Both of them significantly reduced postoperative pain and yielded good cosmesis without increasing other adverse events.

Nonetheless, our method shows some improvements compared to the methods above. Bone cement is easier to obtain than matched iliac crest allografts which are difficult to acquire and carry exorbitant prices, often influencing some patients to refuse the reconstruction. Therefore, the surgery can be carried out in most hospitals. We were able to harvest autologous iliac crest grafts of different sizes and shapes because of the plasticity of bone cement. The surgical procedure is simple and generally takes less time. Compared to the research conducted by Jong Seong Lee et al., we added cancellous screws as a framework to provide an anchor for the bone cement and to prevent displacement and loosening; furthermore, bone cement is not limited to a particular shape.

The causes of postoperative pain after iliac crest harvesting are not clear, although we suspect that postoperative pain may be closely related to the following factors. The process of iliac crest harvesting causes damage to bone and soft tissue, and different sizes of autografts may lead to different degrees of pain [16]. The micromotion of the fracture, stimulation of the bone stump to the soft tissue, and adhesion and scarring of soft tissue are the main causes of postoperative pain. In our study, the decrease in pain may be related to the following factors. Firm fixation of the bone cement is the main reason, which can prevent micromotion of the fracture or between the bone cement and the walls of the bone defect. Bone cement reconstructs the anatomical shape of the iliac crest to reduce irritation to soft tissue. Careful separation of soft tissue and effective stitching can also prevent tissue adhesion and formation of scar.

Reconstruction of iliac crest donor site defects not only alleviates pain but also improves cosmesis and satisfaction. There was no collapse or displacement of implants, which could result in re-operation during the follow-up period, as reported by Jong Seong Lee et al. [12]. We believe that this method of reconstruction is

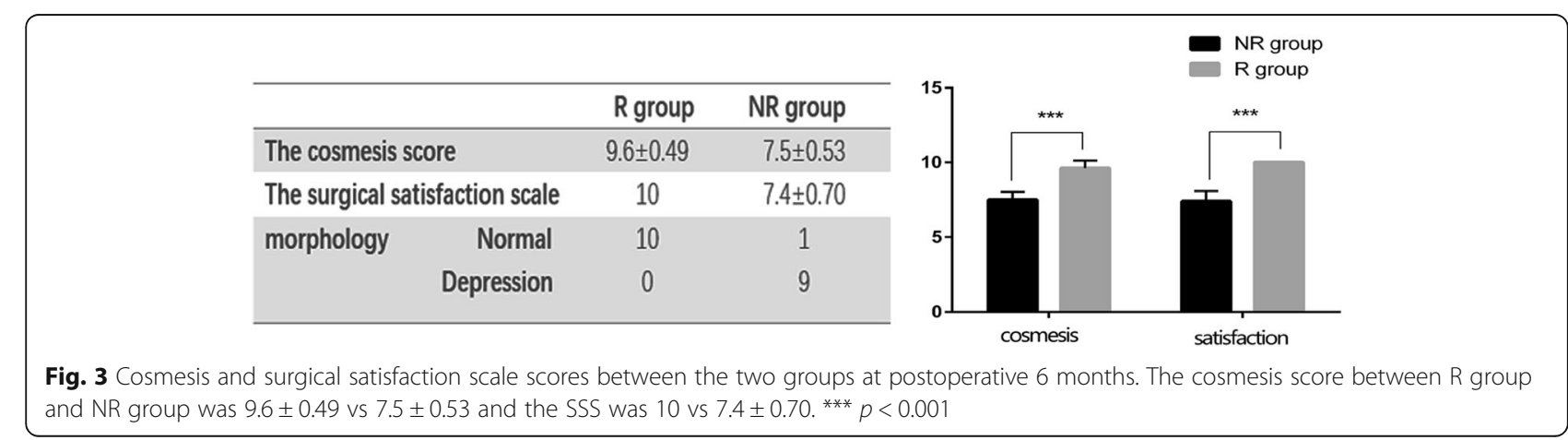




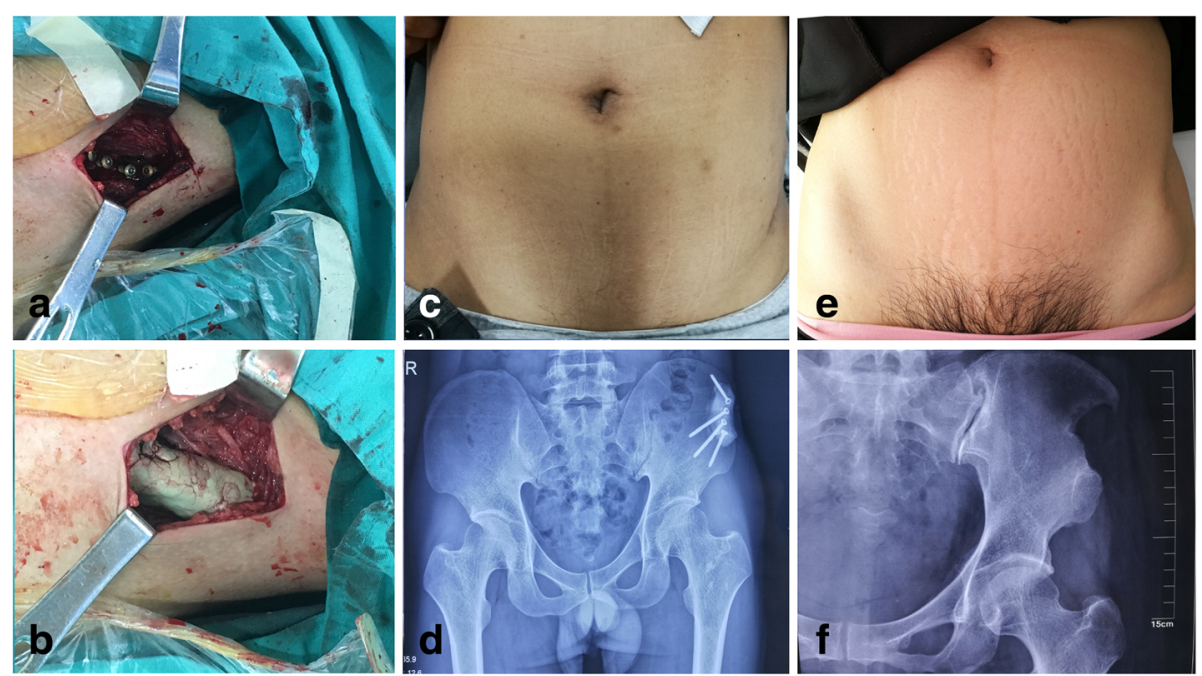

Fig. 4 A 40 years old male $(\mathbf{a}, \mathbf{b}, \mathbf{c}, \mathbf{d})$ in R group and a 47 years old female $(\mathbf{e}, \mathbf{f})$ in NR group, who were diagnosed with giant cell tumor of distal femur, experienced curettage of bone tumor and iliac crest graft. a The screws are implanted after iliac crest harvesting. $\mathbf{b}$ Bone cement is filled into the defect area. $\mathbf{c}$ The cosmesis and morphology of the surgical area compared with contralateral. $\mathbf{d}$ posterior-anterior radiograph images of the pelvis at 6 months after surgical reconstruction. $\mathbf{e}$ and $\mathbf{f}$, The cosmesis of the surgical area compared with contralateral and posterior-anterior radiograph images of the pelvis at 1 year after surgery without reconstruction

more reliable which like a reinforced concrete structure, providing sufficient stability. In addition, this method could prevent some complications such as abdominal hernia theoretically.

This study still has some limitations. Although the results show that patients can benefit from reconstruction of the defect, the sample size was relatively small. Longer follow-up periods may discover additional differences, despite the reported advantages and disadvantages. More careful operation could avoid some complications, such as superficial numbness. In summary, a prospective randomized study with a larger sample and a longer follow-up are necessary. In addition, it should be considered whether pain is associated with the style of surgery that the patient underwent.

\section{Conclusions}

Postoperative pain can be reduced and cosmesis can be improved through reconstructing the iliac crest defects after autogenous harvesting with bone cement and cancellous screws. The technique is simple, safe and easy to be implanted, making it possible to be performed in most hospitals. However, a larger sample of patients and a prospective, controlled study are necessary to verify and extend our results.

\section{Abbreviations}

NR group: The not reconstructed group; pain VAS: The pain visual analog scale; R group: The reconstructed group; SSS: Surgical satisfaction scale

\section{Acknowledgements}

The authors would like to thank all patients who participated in the study.
Funding

No funding was obtained for this study.

\section{Availability of data and materials}

All the data are contained in this paper. The raw data in this study is available from the corresponding author on reasonable request.

\section{Authors' contributions}

Conception and design: ZZ, YD and JZ. Performed the surgery: ZZ.

Development of methodology: JZ, YW and YG. Acquisition of data: JZ and YW. Analysis and interpretation of data: JZ, YW and YG. Writing, review, and/ or revision of manuscript: ZZ, YD, JZ, YW and YG. Study supervision: ZZ, YD. All authors read and approved the final manuscript.

\section{Ethics approval and consent to participate}

This study was in compliance with the Helsinki Declaration. It was approved by the Ethics Committee of Shanghai Jiao Tong University Affiliated Sixth People's Hospital. All participants included in our study were informed and signed the consent.

Consent for publication

Participators agreed to publication and signed the consent.

\section{Competing interests}

The authors declare that they have no competing interest.

\section{Publisher's Note}

Springer Nature remains neutral with regard to jurisdictional claims in published maps and institutional affiliations.

\section{Author details}

'Department of Orthopaedic Surgery, Shanghai Jiao Tong University Affiliated Sixth People's Hospital, Shanghai 200233, China. ${ }^{2}$ Department of Breast Surgery, Key Laboratory of Breast Cancer in Shanghai, Fudan University Shanghai Cancer Center, Fudan University, Shanghai 200032, China. 
Received: 2 August 2017 Accepted: 3 July 2018

Published online: 19 July 2018

\section{References}

1. Zenner J, Hitzl W, Mayer M, Koller H. Analysis of postoperative pain at the anterior iliac crest harvest site: a prospective study of the intraoperative local administration of ropivacaine. Asian Spine J. 2015;9(1):39-46.

2. Kim DH, Rhim R, Li L, Martha J, Swaim BH, Banco RJ, Jenis LG, Tromanhauser SG. Prospective study of iliac crest bone graft harvest site pain and morbidity. Spine J. 2009;9(11):886-92.

3. Armaghani SJ, Even JL, Zern EK, Braly BA, Kang JD, Devin CJ. The evaluation of donor site pain after harvest of Tricortical anterior iliac crest bone graft for spinal surgery: a prospective study. Spine (Phila Pa 1976). 2016;41(4): E191-6.

4. Willcox MJ. Lumbar herniation of kidney following iliac crest bone harvest. Case Rep Surg. 2016;2016:5365647.

5. Fasolis M, Boffano P, Ramieri G. Morbidity associated with anterior iliac crest bone graft. Oral Surg Oral Med Oral Pathol Oral Radiol. 2012;114(5):586-91.

6. Zermatten $P$, Wettstein $M$. Iliac wing fracture following graft harvesting from the anterior iliac crest: literature review based on a case report. Orthop Traumatol Surg Res. 2012;98(1):114-7.

7. Defino HL, Rodriguez-Fuentes AE. Reconstruction of anterior iliac crest bone graft donor sites: presentation of a surgical technique. Eur Spine J. 1999;8(6): 491-4.

8. Chau AM, Xu LL, van der Rijt R, Wong JH, Gragnaniello C, Stanford RE, Mobbs RJ. Reconstruction versus no reconstruction of iliac crest defects following harvest for spinal fusion: a systematic review: a review. J Neurosurg Spine. 2012;16(6):565-72

9. Makridis KG, Ahmad MA, Kanakaris NK, Fragkakis EM, Giannoudis PV. Reconstruction of iliac crest with bovine cancellous allograft after bone graft harvest for symphysis pubis arthrodesis. Int Orthop. 2012;36(8):1701-7.

10. Niu YF, An XF, Wu DJ, Xu SG, Zhang CC, Li M. Anatomical reconstruction of donor site after large iliac crest graft harvest with equivalent iliac crest allograft. A prospective controlled study. Eur Rev Med Pharmacol Sci. 2013; 17(14):1951-7.

11. Burton DC, Carlson BB, Johnson PL, Manna BJ, Riazi-Kermani M, Glattes RC, Jackson RS. Backfilling of iliac crest defects with hydroxyapatite-calcium triphosphate biphasic compound: a prospective, randomized computed tomography and patient-based analysis. Spine J. 2013:13(1):54-61.

12. Lee JS, Park YJ, Wang L, Chang YS, Shetty GM, Nha KW. Modified iliac crest reconstruction with bone cement for reduction of donor site pain and morbidity after open wedge high Tibial osteotomy: a prospective study. Knee Surg Relat Res. 2016;28(4):277-82.

13. O'Neill KR, Lockney DT, Bible JE, Crosby CG, Devin CJ. Bupivacaine for pain reduction after iliac crest bone graft harvest. Orthopedics. 2014;37(5): e428-34.

14. Resnick DK. Reconstruction of anterior iliac crest after bone graft harvest decreases pain: a randomized, controlled clinical trial. Neurosurgery. 2005; 57(3):526-9. discussion 526-529

15. Bapat MR, Chaudhary K, Garg H, Laheri V. Reconstruction of large iliac crest defects after graft harvest using autogenous rib graft: a prospective controlled study. Spine (Phila Pa 1976). 2008;33(23):2570-5.

16. Pirris SM, Nottmeier EW, Kimes S, O'Brien M, Rahmathulla G. A retrospective study of iliac crest bone grafting techniques with allograft reconstruction: do patients even know which iliac crest was harvested? Clinical article. J Neurosurg Spine. 2014;21(4):595-600.

\section{Ready to submit your research? Choose BMC and benefit from:}

- fast, convenient online submission

- thorough peer review by experienced researchers in your field

- rapid publication on acceptance

- support for research data, including large and complex data types

- gold Open Access which fosters wider collaboration and increased citations

- maximum visibility for your research: over $100 \mathrm{M}$ website views per year

At BMC, research is always in progress.

Learn more biomedcentral.com/submissions 